\title{
Secure and Fast Encryption Routine+: Evaluation by
}

\section{Software Application}

\author{
M. Faruk Şahin*, Musaria K. Mahmood*, Indrit Myderrizi* \\ *Department of Electric \& Electronics Engineering, Faculty of Engineering and Architecture, İstanbul Gelisim University, \\ Istanbul, Turkey \\ (m.faruksahin@hotmail.com, mkmahmood@gelisim.edu.tr, $\underline{\text { imyderrizi@gelisim.edu.tr) }}$
}

† Musaria K. Mahmood; Indrit Myderrizi, Tel: +90 21242270 00,

mkmahmood@gelisim.edu.tr

Received: 20.06.2020 Accepted: 24.08.2020

\begin{abstract}
Nowadays, the Internet era and its components are based on the collection, exchange, and storage of information which represent a sever security concern. Many cipher algorithms have been developed, evaluated, and implemented to increase the protection of data for various applications. The most known strategy of data securing has been the implementation of symmetrical key encryption standards. Secrecy, robustness, reliability, and software/ hardware implementation, are among the most important properties of good cipher standard. In this work, text, data and image encryption/decryption are performed by a MATLAB software implementation of the symmetric key block standard realized using the Secure and Fast Encryption Routine (SAFER+) algorithm with 128 key length option. The results show good performances in encryption/ decryption speed through a set of original procedures to simplify the achievement of nonlinear functions adopted by SAFER+ structure.
\end{abstract}

Keywords- SAFER+; data security; MATLAB; cryptology;

\section{Introduction}

The rapid development of data transmission in various fields including education, banking, military, industrial and many other fields has made cryptography and data security as the main stone in all information systems. The evulotion of the Internet toward the Internet of Thing (IoT), and The Internet of Everything (IoE) has promoted the need of secure and delay-free communication $[1,2]$. Information and confidentiality has become one of the most important and urgent requirements in today's systems. Various encryption algorithms developed through the science of cryptography are used today for securing data exchange between different 
INTERNATIONAL JOURNAL of ENGINEERING TECHNOLOGIES-IJET

Şahin, et al, Vol.6, No.2, 2020

parties. The encryption systems fall into two categories, the asymmetric and symmetric key encryption standards [3]. The asymmetric encryption algorithms provide two different keys, one for encryption usually available and known by all parties and the second key for decryption which is known by the owner and kept secret. The encryption key is the public key while that used for decryption is the private key. DiffieHelman, RSA are examples of asymmetric encryptions [4]. Symmetric encryption algorithms use one secret key shared by both parties (sender and receiver) for data encryption/ decryption. Along with the secret texts exchange, the symmetrical keys must also be updated every predefined period of time through securet sharing system usually based on asymerical key standard. The plaintext (data) and the symmetric key are the inputs of the system while the encrypted message is the output. In the receiver side the reverse process is planned by injecting the encrypted data with the same key into the decryption system to reproduce the original plaintext. Many symmetrical encryption standards exist today such as the Data Encryption Standard (DES), Triple Data Encryption Standard (3DES) [4], Blowfish [5], Secure And Fast Encryption Routine (SAFER+) [6], and the Rijndael which is selected by the U.S. National Institute of Standards and Technology (NIST) as the actual Advanced Encryption Standard (AES) since 2001 [7, 8]. Symmetric key encryption standards are divided into two types; the block and stream encryption algorithms. The block ciphers encrypt/ decrypt fixed long length blocks of data, whereas the stream cipher is operating by combining plaintext digits with a pseudorandom keystream resulting on encryption of short data characters. One of the most important advantages of symmetric key

encryption is its high speed compared to the asymmetric key encryption, which make it suitable for real time applications. Added to that, it is much easier to implement the symmetric key algorithm on hardware using digital electronic gates based on the repetition of elementary mathematical operations [9]. SAFER + is the new algorithm of the SAFER family designed by James Messey in Cylink. It is one of the symmetric key algorithms known by its performance in data encryption speed that makes it suitable for real-time data encryption requirements. SAFER+ offers high efficiency and low memory requirement compared to other algorithms [10]. SAFER+ is the most used encryption standard in the Bluetooth security architecture. The confrontation of SAFER+ standard with existing encryption algorithms proves its superiority in Bluetooth algorithms, but this encryption system is also used in other fields [11]. Its digital VLSI design and FPGA implementation shows an outstanding performance in terms of minimization of delay which enables this algorithm to be utilized for high-speed applications [12, 13]. The software implementation of the algorithm confirms its speed quality for various case studies including speech, image and data in general. While moving from theory to practice, the parts that are hard to handle in software implementation are nonlinear functions that must be solved by inventory procedure [14].

\section{SAFER+ encryption/ decryption philosophy}

\subsection{General}

SAFER encryption/ decryption family is byte oriented cipher algorithm acting over a predefined data block size which is a very advantageous situation for 8 bit microprocessors. SAFER+ is a block cipher encrypting 16-byte (128 bits) data blocks through a number of encryption/decryption cycles. The 


\section{Şahin, et al, Vol.6, No.2, 2020}

number of encryption/ decryption cycles is connected to the key lengths which comes in three options [10]:

- Key length equal to 128 bits yields an encryption/ decryption processes with $R=8$ rounds,

- Key length equal to 192 bits yields an encryption/ decryption processes with $R=12$ rounds,

- Key length equal to 256 bits yields an encryption/ decryption processes with $R=16$ rounds.

Starting from the original secrete symmetric key $\left(K_{1}\right)$ as input, a group of $2 R$ keys $\left(K_{2} \ldots K_{2 R+1}\right)$ is generated, each with the same length as the original key, by the key schedule subroutine. SAFER+ is composed by many encryption/ decryption layers (linears and nonlinears) based on byte addition, bit addition, logarithm, exponential, matrix multiplication, bits rotating, and many other funtions and procedures. In this research paper, the SAFER+ cipher algorithm with the option of 128-bit key length is adopted.

\subsection{Additive groups}

The group operations used in the encryption/ decryption rounds consists of the interaction between the subkeys and a 16-byte data groups. Eight of data groups are subject to normal arithmetic byte addition modulo 256 (add) while the other eight bytes are bit-by-bit added modulo 2 with their peer bytes in the subkeys (xor). Note that for the decryption rounds, a byte- by-byte subtraction function (sub) is used instead of the add function to reverse the data generation process. Implementing two different group operations between the subkeys and the data increases the degree of robustness of the cipher protocol by consolidating the randomness and the ambiguity of the resulting ciphertext. The add/sub, and xor operations are performed two-by-two in a sequential manner, and in the opposite locations for the decryption process compared to the encryption process. At each encryption round, two subkeys are used in two consecutive encryption layers, where the bit-by-bit modulo 2 and byte-addition modulo 256 operations take place between subkeys and data as depicted in the Fig. 1.

\subsection{Nonlinear layers: Exponential and Logarithmic functions}

A nonlinear encryption layer is used at each encryption/ decryption round based on two anti-functions; the exponential and the logarithmic functions. It is the most difficult part in the program implementation due to the limitation of software tools when dealing with big numbers exceeding their memory limits. This constraint is resolved in this work by providing an original procedure called the Exponential Logarithmic Function (ELF) which is used to resolve the mathematical calculations. ELF operates at each round in the encryption/ decryption procedures between the two additive layers. The exponential and logarithmic fuctions in the ELF are defined according to the base 45 such that:

\section{$\underline{\text { Exponential function }}$}

For $0 \leq x \leq 255$, we have:

$$
F(x)= \begin{cases}45^{x} \bmod 257 & \text { if } x \neq 128 \\ 0 & \text { if } x=128\end{cases}
$$

\section{$\underline{\text { Logarithmic function }}$}

For $0 \leq y \leq 255$, we have:

$$
G(y)= \begin{cases}\log _{45}(y) & \text { if } y \neq 0 \\ 128 & \text { if } y=0\end{cases}
$$

It is proven that the choice of exponential and logarithmic as the two mutually inverse functions in this algorithm is a distinctive choice because of substantial fraction of all mutually inverse nonlinear functions when used in their place 
INTERNATIONAL JOURNAL of ENGINEERING TECHNOLOGIES-IJET

Şahin, et al, Vol.6, No.2, 2020

would lead to recognize plaintext attack faster than exhaustive search [10].

\subsection{Invertible linear transformation}

At the end of every encryption round, a block of linear

transformation by matrix multiplication is used by multiplying the output of the second additive layer and the matrix M. M is an invertible predefined $16 \times 16$ matrix used for encryption while its inverse matrix $\mathrm{M}^{-1}$ is used in the decryption process. At the output of the second additive layer, the 16 bytes are multiplied by the matrix $\mathrm{M}$ in $\bmod 256$ arithmetic that generates the round data output. The matrix $\mathrm{M}$ and $\mathrm{M}^{-1}$ are given by the equations (3) and (4):
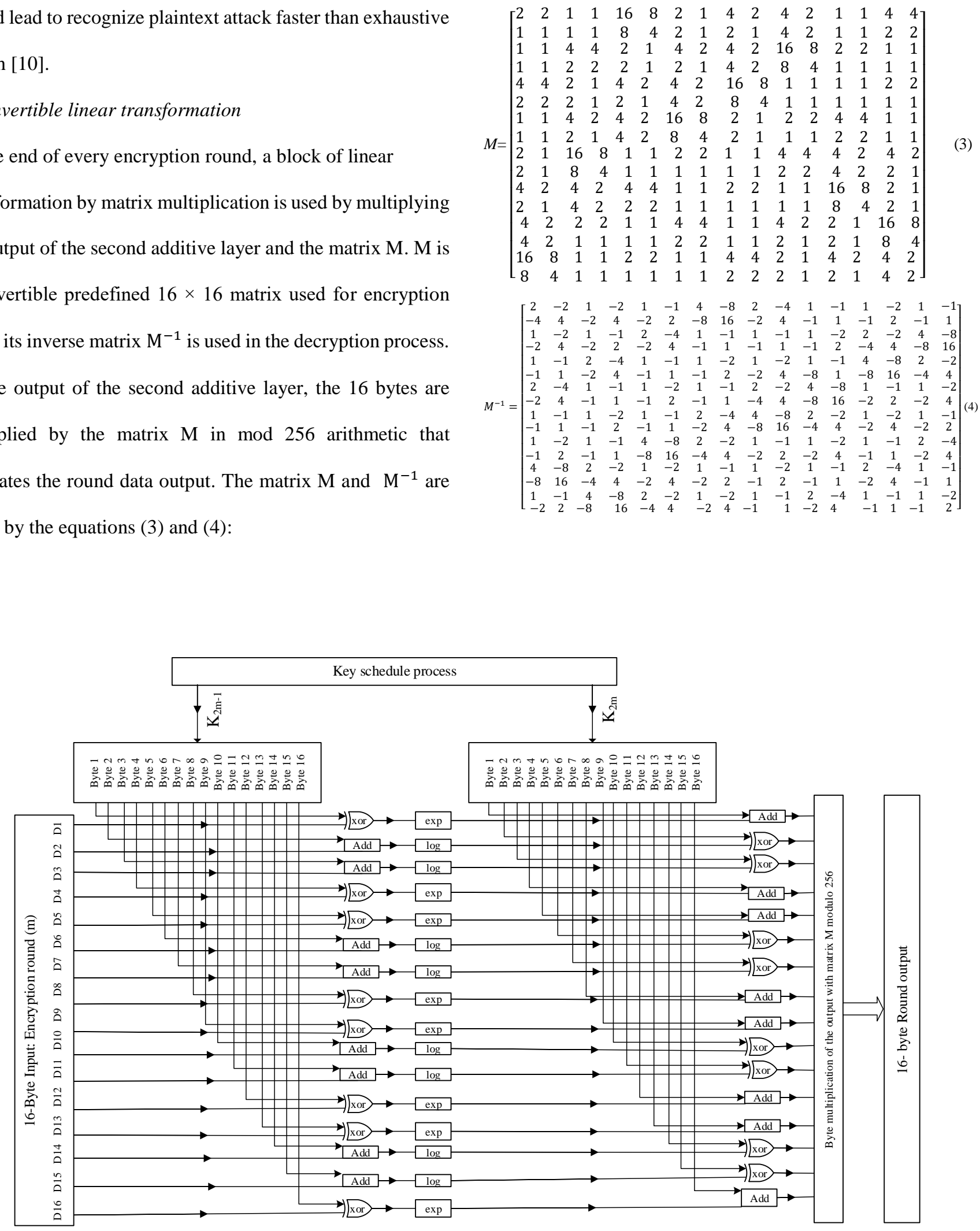

Figure 1. Digital Design and structure of the Encryption Rounds (m) 
The negative elements in the matrix $\mathrm{M}^{-1}$ vanish by the modulo 256 operations of the matrix giving a matrix with all element positives. The composition of the matrix $M$ is a noticeable improvement compared to the matrices used for the same linear transformation layer in the other SAFER family standards. There are at least five elements in each row of $\mathrm{M}$ with their value equal to one which means that an input 16byte vector of one element different to zero will generate output block with at least five non-zero bytes. This yields a very fast diffusion property by the matrix $\mathrm{M}$ and contributes highly to improve the resistance against differential cryptanalysis. If the 16-byte vector $B T=\left[b t_{1}, b t_{2}, \ldots, b t_{16}\right]$, is post multiplied by $M\left[a_{i j}\right]$, the output $B O$ is a 16-byte vector:

$B O=B T \times M=\left[b o_{1}, b o_{2}, \ldots, b o_{16}\right]$

$b o_{k}=\left\{\sum_{i=1}^{16} b t_{i} \times a_{k i}\right\} \bmod 256$

where $B O$ is the 16-byte vector output from an encryption round. The inverse linear transformation is performed by a similar matrix multiplication process at the beginning of each decryption round between the 16 -byte input vector and $M^{-1}$ as presented in the Fig. 2 .

\subsection{Bias Matrix: $B$}

Starting from the secret key $K_{1}$ carried by the two communicating parties, SAFER+ generates the group of $2 R$ subkeys $\left(K_{2}, K_{3}, \ldots, K_{2 R+1}\right)$ required for the $R$ encryption/ decryption rounds. The key schedule utilizes 17 words, each composed by 16 byte elements that can be presented in a precalculated matrix, called the bias matrix given as in the equation (7).

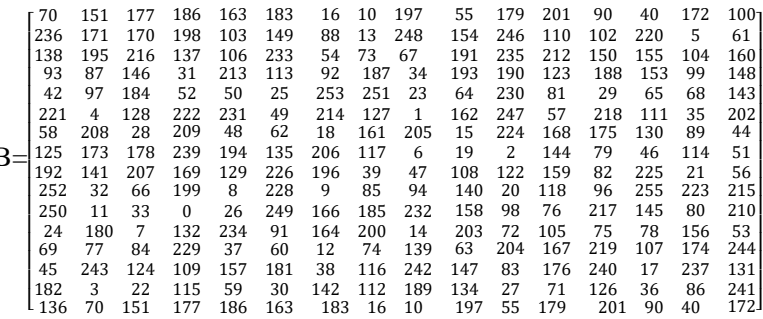

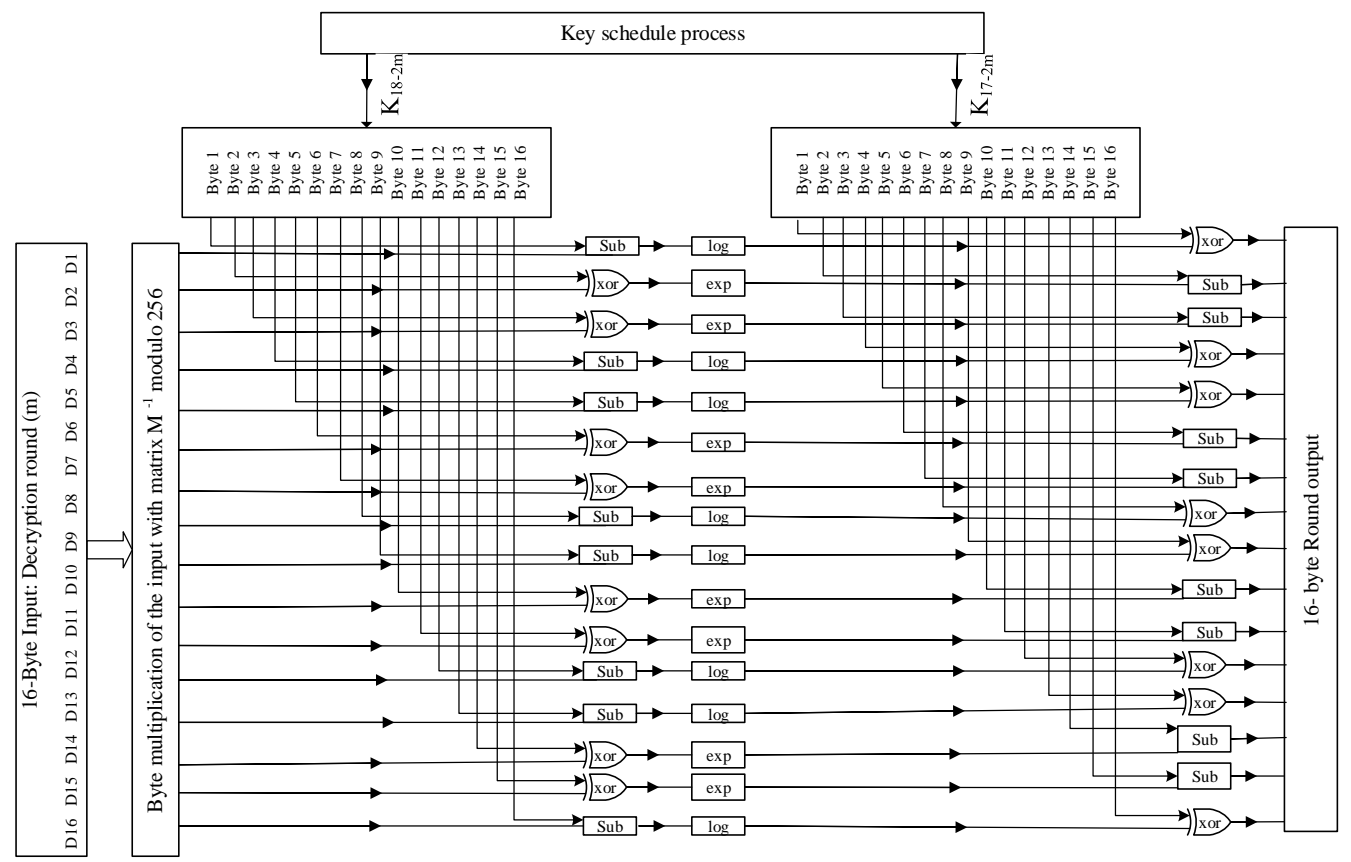

Figure 2. Digital Design of the Decryption Rounds Structure 
INTERNATIONAL JOURNAL of ENGINEERING TECHNOLOGIES-IJET

\section{Şahin, et al, Vol.6, No.2, 2020}

Bias matrix is used to randomize the key schedules and is composed of a number of rows equal to the subkeys number (including $K_{1}$ ), thus $2 R+1$ rows and $2 R$ columns. The first row is a dummy one because the key $K_{1}$ already exists, so it is not included in the matrix given in Eq (7). The bias matrix $B\left[b_{i j}\right]$ is generated by the formula:

$b_{i j}=45^{\left(45^{17 i+j} \bmod 257\right)} \bmod 257$

where by definition $\mathrm{Eq}(8)$ gives a result equal to 256, if the $b_{i j}$ is set to zero. For the option adopted by this work with a key equal to 16 bytes, $i=[2,3, \ldots, 17]$, and $j=[1,2, \ldots, 16]$.

\section{Encryption/ Decryption Subroutines}

\subsection{Key Schedules}

The strongness of a symetrical key encryption algorithm is accomplished through the structure of encryption/ dectryption as well as by the complexity of generating the group of subkeys $\left(K_{2}, \ldots, K_{17}\right)$ by the key schedules. A total of 17 keys of 128-bit (16 bytes) are created from the symmetric key $\left(K_{1}\right)$ by various mathematical operations with the randomness of keys group in mind as shown in the Fig. 3.

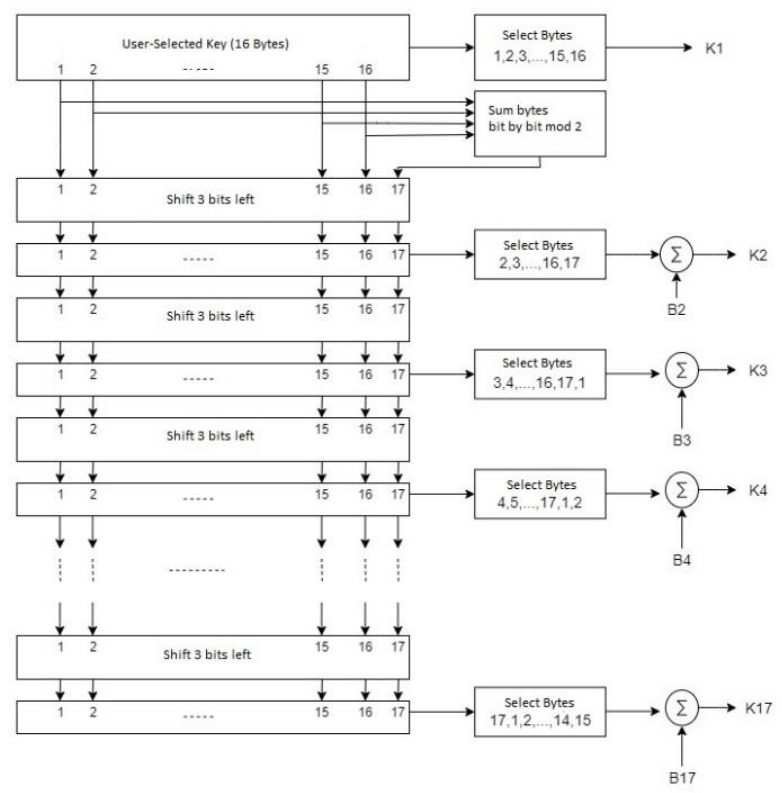

Figure 3. 128-bit Keys production
The 16 byte words input key $\left(K_{1}\right)$ are added bit-by-bit modulo 2 to calculate the byte number 17 which is used in the next operations. Subkeys $\left(K_{2}, \ldots, K_{17}\right)$ are calculated sequentially based on three operations for each subkey, they are bits shifting, byte selection, permutation and addition with the bias vector. The production of the subkey $\left(K_{n}\right)$ starts by shifting three bits to the left for each byte in order to generate a new bits patern. A selection process follows where 16-byte vector $\left(B t_{1}, \ldots, B t_{16}\right)$ is selected among the 17 bytes sink where the byte number $(n-1)$ is neglected. The permutation of byte words changes their order in the vector such that the vector becomes $\left(B t_{n}, \ldots, B t_{17}, B t_{1}, \ldots, B t_{n-1}\right)$. Finally, the key $\left(K_{n}\right)$ is the result of byte addition modulo 256 of the vector $\left(B t_{n}, \ldots, B t_{17}, B t_{1}, \ldots, B t_{n-1}\right)$ and the bias vector $B_{n}$ (row $n$ from matrix $B$ ).

\subsection{Encryption Procedure}

The general structure of the encryption procedure for 128-bit key length comprises eight encryption rounds and one encryption output layer as presented in the Fig. 4.

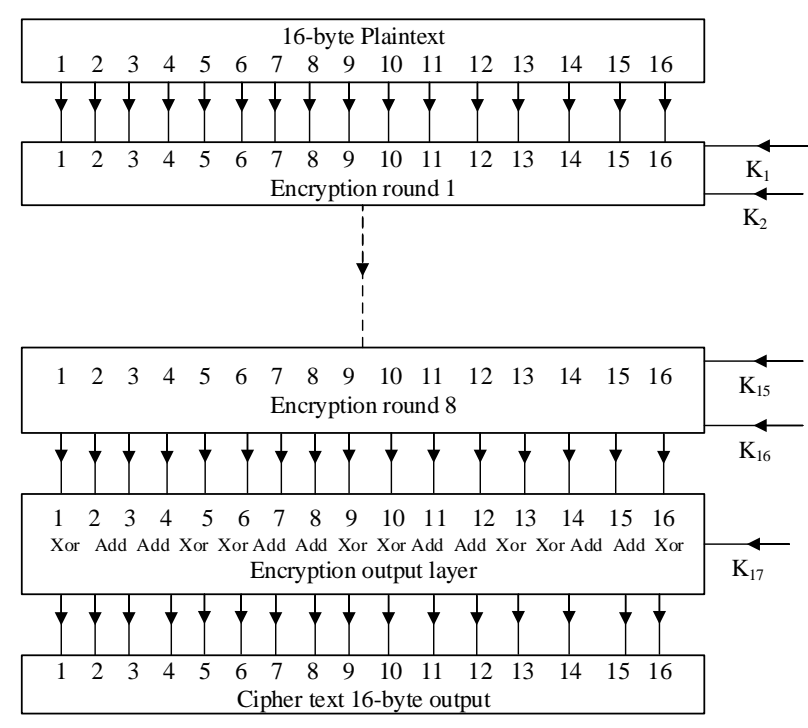

Figure 4: Encryption Structure 


\section{Şahin, et al, Vol.6, No.2, 2020}

There are two kinds of input to this procedure, the plaintext (128 bits) and the subkeys group $\left(K_{1}, \ldots, K_{17}\right)$. The input plaintext is subject to a sequence of eight encryption cycles comprising the same mathematical linear and nonlinear functions where two keys from the group $\left(K_{1}, K_{2}, \ldots, K_{16}\right)$ are used at each round as shown in the Fig. 5. The last key $K_{17}$ is then used for the output encryption stage. At the round (m), the 16 bytes input data are advanced to the first additive layer such that byte ( $\mathrm{j}$ ) is bit-by-bit modulo 2 added to its peer byte in the subkey $K_{2 m-1}$ for $\mathrm{j}=1,4,5,8,9,12,13$, and 16. The remaining bytes are then byte-by-byte modulo 256 added with their peer byte of the same subkey for $j=2,3,6,7,10,11,14$, and 15. This process is repeated in reverse partition for the second additive layer in the same round but with the subkey $K_{2 m}$ as presented in the Fig. 1. The output of the first additive layer is processed by the nonlinear layer such that when bitby-bit addition is used, the nonlinear operation is the exponential, while the logarithmic function is used for data coming from byte-by-byte addition.

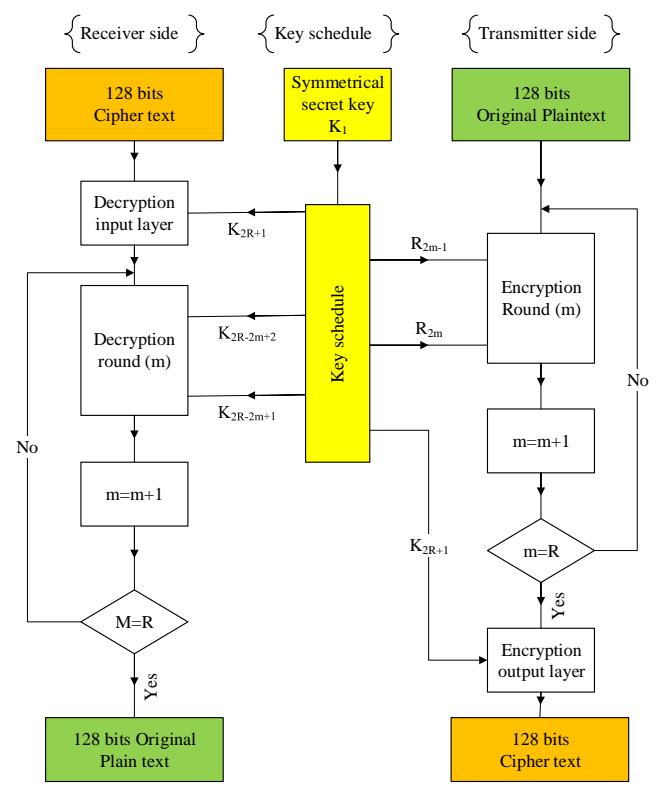

Figure 5 : Encryption/decryption structure
The output of the nonlinear layer is interacted with $K_{2 m}$ in the second additive layer, and then by the linear transformation of matrix multiplication with $\mathrm{M}$. The output of the round (m) is send to the input of the round $(m+1)$ as shown in the Fig. 4 . After completing 8 encryption cycles the output is forwarded to the output encryption layer, where the same operations stated in the first additive layer are performed with the last subkey $\left(K_{17}\right)$ as input, resulting in the final ciphertext block output.

\subsection{Decryption Procedure}

The decryption subroutine is composed by the inverse mathematical operations, and starts with the input decryption stage using $K_{17}$, followed by the same number of successive rounds $R=8$ where two subkeys are used at each round as presented in Fig. 6. Ciphertext block at the receiver side is forwarded to the input decryption stage where bytes located at the positions $j=\{1,4,5,8,9,12,13,16\}$ are bit-by-bit modulo 2 added with their peer bytes of the key $K_{17}$, whereas

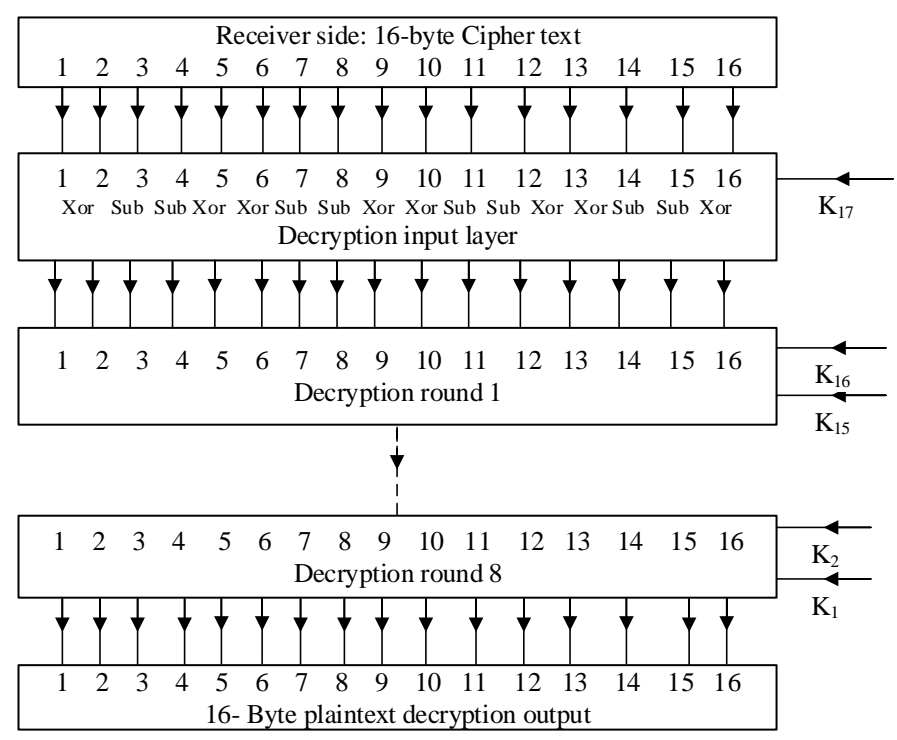

Figure 6. Decryption Structure 


\section{Şahin, et al, Vol.6, No.2, 2020}

the remainding bytes at the positions $j=\{2,3,6,7,10,11,14$,

$15\}$ of the subkey are subtracted modulo 256 from their peer

ciphertext bytes. The the data proceeded to the first decryption

cycle where the subkeys $K_{16}$ and $K_{15}$ are used. The decryption process is the opposite of encryption, in that, the subkeys allocation for successif cycles are done in decreasing order starting from $K_{16}$ to $K_{1}$. Although the bit-by-bit modulo 2 operation (xor) is still used in the same manner, the byte-bybyte addition is replaced by the byte-by-byte subtraction. The decryption round $(\mathrm{m})$ begins by the multipling its 16-byte input vector by the matrix $M^{-1}$ modulo 256 . The resulting vector is then directed to the first additive layer in the decryption cycle where byte at the positions $j=$ $\{2,3,6,7,10,11,14,15\}$ are bit-by-bit modulo 2 added with their peer bytes of the subkey $\left(K_{18-2 m}\right)$ while the bytes at the positions $j=\{1,4,5,8,9,12,13,16\}$ of the subkey are subtracted from their peer bytes in the input vector modulo 256, with all their values between 0 and 255 . The output of this layer is directed to the nonlinear layer where the exponential fuction is applied for byte resulting from xor operations while a logarithm function is used for byte issued from subtraction. The result from the decryption cycle (m) is the output of the second additive layer which is applied with the reverse operations compared to the first additive layer. This process is repeated for 8 decryption cycles yielding at the end to the regeneration of the original plaintext.

\section{Simulation Methology and Results}

\section{1 linear layers implementation}

The simulation of SAFER+ is performed by MATLAB portal where the principle logic functions such as bytes addition, bit addition, modulo operation, matrix multiplication, bits rotation, and byte selection exist as in-program functions.

\section{2 non-linear layer implementation}

The most difficult programming step in SAFER+ is the implementation of ELF due to the huge numbers resulting from the exponential function which are hard to be handled by any programming language. After careful examination of the exponential function, the MATLAB is found to produce correct results for $45^{n}$ if $0 \leq n \leq 8$, but yet the results become erroneous for $n \geq 9$. To prevent this, the ELF procedure is developped to overcome the programming limitations and to evaluate correctly the output function of the exponential stage $F(x)$ as shown in Fig. 7. Since $r<8$, a correct evaluation of $F(x)$ for all values of $0 \leq x \leq 255$ is obtained. After that the last stage of the procedure yields to the correct result. The logarithmic and exponential are reverse functions, so the transformation table of the logarithm can be deduced from that of the exponential by the reciprocity of values yielding to the accomplishement of the whole ELF procedure:

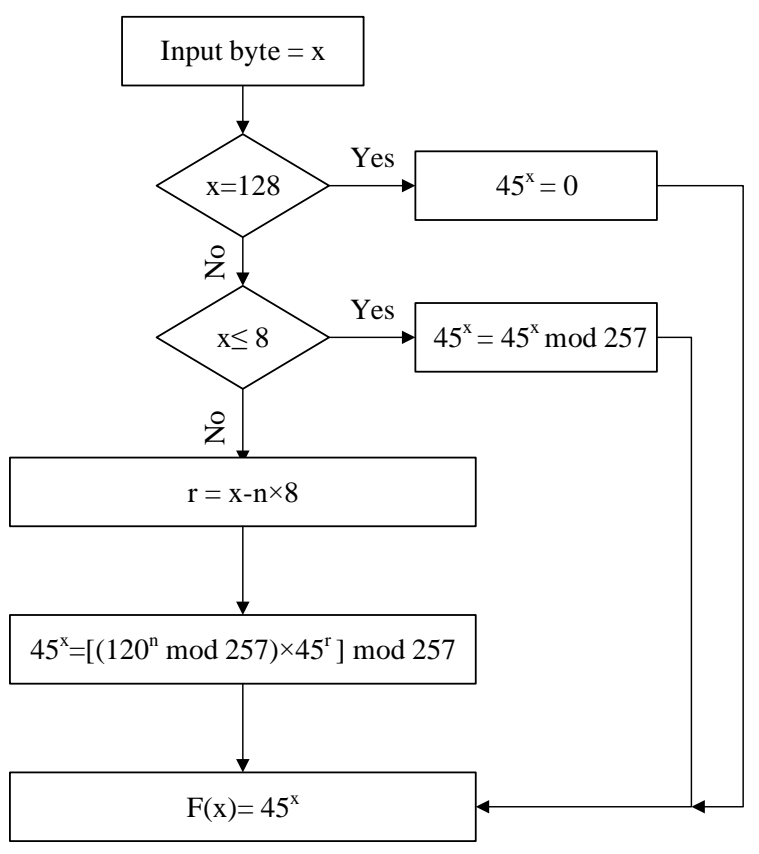

Figure 7. Exponential function implementation (ELF) 
Şahin, et al, Vol.6, No.2, 2020

- If $F(x)=45^{x}=y$, then $\log _{45}(y)=x$

- If $y=0$, then $\log _{45}(0)=128$.

The application of the ELF gives the exponential transformation table for $x=[0,1, \ldots, 255]$, and $F(x)$ is evaluated to be:

$\left[\begin{array}{lllllllllll}1 & 45 & 226 & 147 & 190 & 69 & 21 & 174 & 120 & 3 & 135\end{array}\right.$ $\begin{array}{llllllllllll}164 & 184 & 56 & 207 & 63 & 8 & 103 & 9 & 148 & 235 & 38 & 168\end{array}$ $\begin{array}{lllllllllll}107 & 189 & 24 & 52 & 27 & 187 & 191 & 114 & 247 & 64 & 53\end{array}$

$\begin{array}{lllllllllll}72 & 156 & 81 & 47 & 59 & 85 & 227 & 192 & 159 & 216 & 211\end{array}$

$\begin{array}{llllllllll}243 & 141 & 177 & 255 & 167 & 62 & 220 & 134 & 119 & 215\end{array}$

$\begin{array}{llllllllll}166 & 17 & 251 & 244 & 186 & 146 & 145 & 100 & 131 & 241\end{array}$

$\begin{array}{lllllllllll}51 & 239 & 218 & 44 & 181 & 178 & 43 & 136 & 209 & 153 & 203\end{array}$

$\begin{array}{lllllllllll}140 & 132 & 29 & 20 & 129 & 151 & 113 & 202 & 95 & 163 & 139\end{array}$

$\begin{array}{llllllllllll}87 & 60 & 130 & 196 & 82 & 92 & 28 & 232 & 160 & 4 & 180 & 133\end{array}$

$\begin{array}{lllllllllll}74 & 246 & 19 & 84 & 182 & 223 & 12 & 26 & 142 & 222 & 224\end{array}$

$\begin{array}{lllllllllll}57 & 252 & 32 & 155 & 36 & 78 & 169 & 152 & 158 & 171 & 242\end{array}$

$\begin{array}{lllllllllll}96 & 208 & 108 & 234 & 250 & 199 & 217 & 0 & 212 & 31 & 110\end{array}$

$\begin{array}{lllllllllll}67 & 188 & 236 & 83 & 137 & 254 & 122 & 93 & 73 & 201 & 50\end{array}$

$\begin{array}{lllllllllll}194 & 249 & 154 & 248 & 109 & 22 & 219 & 89 & 150 & 68 & 233\end{array}$

$\begin{array}{lllllllllll}205 & 230 & 70 & 66 & 143 & 10 & 193 & 204 & 185 & 101 & 176\end{array}$

$\begin{array}{llllllllllll}210 & 198 & 172 & 30 & 65 & 98 & 41 & 46 & 14 & 116 & 80 & 2\end{array}$

$\begin{array}{llllllllllll}90 & 195 & 37 & 123 & 138 & 42 & 91 & 240 & 6 & 13 & 71 & 111\end{array}$

$\begin{array}{lllllllllll}112 & 157 & 126 & 16 & 206 & 18 & 39 & 213 & 76 & 79 & 214\end{array}$

$\begin{array}{lllllllllll}121 & 48 & 104 & 54 & 117 & 125 & 228 & 237 & 128 & 106 & 144\end{array}$

$\begin{array}{lllllllllll}55 & 162 & 94 & 118 & 170 & 197 & 127 & 61 & 175 & 165 & 229\end{array}$

$\begin{array}{lllllllllll}25 & 97 & 253 & 77 & 124 & 183 & 11 & 238 & 173 & 75 & 34\end{array}$

$\begin{array}{lllllllllll}245 & 231 & 115 & 35 & 33 & 200 & 5 & 225 & 102 & 221 & 179\end{array}$

$\begin{array}{llllllllllll}88 & 105 & 99 & 86 & 15 & 161 & 49 & 149 & 23 & 7 & 58 & 40]\end{array}$

where $F(0)=1, F(1)=45, \ldots, F(255)=40$.

From the above transformation table the logarithmic tranformation table can be deduced such that if:
$F(x)=45^{x}=y$, then $G(y)=\log _{45}(y)=x$. This gives the following logarithm transformation table:

$\left[\begin{array}{lllllllllll}128 & 0 & 176 & 9 & 96 & 239 & 185 & 253 & 16 & 18 & 159\end{array}\right.$ $\begin{array}{lllllllllll}228 & 105 & 186 & 173 & 248 & 192 & 56 & 194 & 101 & 79 & 6\end{array}$ $\begin{array}{lllllllllll}148 & 252 & 25 & 222 & 106 & 27 & 93 & 78 & 168 & 130 & 112\end{array}$ $\begin{array}{llllllllll}237 & 232 & 236 & 114 & 179 & 21 & 195 & 255 & 171 & 182\end{array}$ $\begin{array}{llllllllllll}71 & 68 & 1 & 172 & 37 & 201 & 250 & 142 & 65 & 26 & 33 & 203\end{array}$ $\begin{array}{lllllllllll}211 & 13 & 110 & 254 & 38 & 88 & 218 & 50 & 15 & 32 & 169\end{array}$ $\begin{array}{lllllllllll}157 & 132 & 152 & 5 & 156 & 187 & 34 & 140 & 99 & 231 & 197\end{array}$ $\begin{array}{lllllllllll}225 & 115 & 198 & 175 & 36 & 91 & 135 & 102 & 39 & 247 & 87\end{array}$ $\begin{array}{lllllllllll}244 & 150 & 177 & 183 & 92 & 139 & 213 & 84 & 121 & 223 & 170\end{array}$ $\begin{array}{lllllllllll}246 & 62 & 163 & 241 & 17 & 202 & 245 & 209 & 23 & 123 & 147\end{array}$ $\begin{array}{lllllllllll}131 & 188 & 189 & 82 & 30 & 235 & 174 & 204 & 214 & 53 & 8\end{array}$ $\begin{array}{lllllllllll}200 & 138 & 180 & 226 & 205 & 191 & 217 & 208 & 80 & 89 & 63\end{array}$ $\begin{array}{llllllllllll}77 & 98 & 52 & 10 & 72 & 136 & 181 & 86 & 76 & 46 & 107 & 158\end{array}$ $\begin{array}{llllllllllll}210 & 61 & 60 & 3 & 19 & 251 & 151 & 81 & 117 & 74 & 145 & 113\end{array}$ $\begin{array}{lllllllllll}35 & 190 & 118 & 42 & 95 & 249 & 212 & 85 & 11 & 220 & 55\end{array}$ $\begin{array}{lllllllllll}49 & 22 & 116 & 215 & 119 & 167 & 230 & 7 & 219 & 164 & 47\end{array}$ $\begin{array}{lllllllllll}70 & 243 & 97 & 69 & 103 & 227 & 12 & 162 & 59 & 28 & 133\end{array}$ $\begin{array}{lllllllllll}24 & 4 & 29 & 41 & 160 & 143 & 178 & 90 & 216 & 166 & 126\end{array}$ $\begin{array}{lllllllllll}238 & 141 & 83 & 75 & 161 & 154 & 193 & 14 & 122 & 73 & 165\end{array}$ $\begin{array}{lllllllllll}44 & 129 & 196 & 199 & 54 & 43 & 127 & 67 & 149 & 51 & 242\end{array}$ $\begin{array}{lllllllllll}108 & 104 & 109 & 240 & 2 & 40 & 206 & 221 & 155 & 234 & 94\end{array}$ $\begin{array}{lllllllllll}153 & 124 & 20 & 134 & 207 & 229 & 66 & 184 & 64 & 120 & 45\end{array}$ $\begin{array}{lllllllllll}58 & 233 & 100 & 31 & 146 & 144 & 125 & 57 & 111 & 224 & 137\end{array}$ 48].

\subsection{Data Block Encryption/ decryption}

A data block of 16 bytes is used as plaintext to evaluate the correcteness of the encryption/ decryption SAFER+ implementation. A user key $K_{1}$, is given also as input, the subroutine key schedules is generating using this key the 
INTERNATIONAL JOURNAL Of ENGINEERING TECHNOLOGIES-IJET

Şahin, et al, Vol.6, No.2, 2020

group of 17 subkeys $\left(K_{1}, K_{2}, \ldots, K_{17}\right)$. Based on the input

plaintext and the user key, the ciphertext is the result of the

SAFER+ algorithm as shown in the result table 1 . The

decryption process is evaluated through the same key $K_{1}$ and

with the collected ciphertext vector as input. The resulting

vector is the same as the plaintext used as input for the

encryption process which validates the exactness of the

software implementation of SAFER+ algorithm and its three

components; the encryption, the decryption, and the key

schdules subroutines. SAFER+ is known by its property as

high speed encryption procedure which enables it to be choosen for Bluetooth communication among other applications. The simulation is performed using a personal computer Intel Core i5 7300HQ, 2.5 GHz, 8 GB DDR4 RAM, $2400 \mathrm{MHz}$, where the encryption process for one data block of 16 bytes is done in $0.063131 \mathrm{sec}$, and the decryption process in $0.026952 \mathrm{sec}$. This performs better compared to the MATLAB implementation of the AES in [8], where at least $0.088 \mathrm{sec}$ is needed for the encryption of 16 bytes by a computer with similar features. The decryption process in the presented work consumes less time due to the simplifications of the logarithmic function evaluation through the ELF procedure.

Table 1: Simulation results

\begin{tabular}{|c|c|c|c|c|c|c|c|c|c|c|c|c|c|c|c|c|c|}
\hline Input & Plaintext & 179 & 166 & 219 & 60 & 135 & 12 & 62 & 153 & 36 & 94 & 13 & 28 & 6 & 183 & 71 & 222 \\
\hline Input & $K_{1}$ & 41 & 35 & 190 & 132 & 225 & 108 & 214 & 174 & 82 & 144 & 73 & 241 & 241 & 187 & 233 & 235 \\
\hline Output & $K_{2}$ & 295 & 140 & 213 & 201 & 6 & 109 & 133 & 156 & 73 & 129 & 66 & 88 & 55 & 119 & 11 & 35 \\
\hline Output & $K_{3}$ & 155 & 204 & 34 & 225 & 28 & 64 & 236 & 49 & 74 & 22 & 144 & 92 & 224 & 214 & 2 & 135 \\
\hline Output & $K_{4}$ & 147 & 134 & 176 & 54 & 199 & 141 & 87 & 219 & 38 & 162 & 98 & 167 & 109 & 138 & 186 & 230 \\
\hline Output & $K_{5}$ & 123 & 29 & 255 & 9 & 250 & 122 & 240 & 218 & 65 & 124 & 92 & 57 & 59 & 43 & 149 & 127 \\
\hline Output & $K_{6}$ & 96 & 204 & 15 & 93 & 122 & 189 & 245 & 243 & 244 & 52 & 219 & 76 & 177 & 210 & 163 & 209 \\
\hline Output & $K_{7}$ & 56 & 190 & 201 & 32 & 12 & 248 & 157 & 109 & 168 & 81 & 214 & 221 & 102 & 105 & 53 & 81 \\
\hline Output & $K_{8}$ & 15 & 26 & 46 & 250 & 110 & 124 & 137 & 222 & 74 & 13 & 5 & 12 & 134 & 18 & 149 & 185 \\
\hline Output & $K_{9}$ & 207 & 61 & 251 & 224 & 179 & 66 & 183 & 96 & 253 & 60 & 37 & 78 & 211 & 15 & 222 & 9 \\
\hline Output & $K_{10}$ & 68 & 215 & 94 & 56 & 94 & 49 & 35 & 230 & 120 & 133 & 111 & 195 & 97 & 68 & 203 & 173 \\
\hline Output & $K_{11}$ & 78 & 156 & 190 & 181 & 130 & 222 & 6 & 159 & 38 & 59 & 53 & 238 & 123 & 180 & 138 & 107 \\
\hline Output & $K_{12}$ & 221 & 238 & 152 & 211 & 241 & 232 & 248 & 255 & 101 & 167 & 37 & 36 & 134 & 238 & 244 & 243 \\
\hline Output & $K_{13}$ & 55 & 111 & 165 & 66 & 105 & 237 & 214 & 179 & 86 & 233 & 14 & 214 & 53 & 115 & 165 & 201 \\
\hline Output & $K_{14}$ & 34 & 65 & 73 & 224 & 185 & 205 & 107 & 140 & 123 & 117 & 55 & 254 & 4 & 179 & 82 & 236 \\
\hline Output & $K_{15}$ & 212 & 162 & 91 & 17 & 41 & 175 & 56 & 251 & 163 & 238 & 13 & 249 & 50 & 54 & 180 & 74 \\
\hline Output & $K_{16}$ & 51 & 1 & 59 & 215 & 18 & 174 & 202 & 253 & 151 & 91 & 101 & 89 & 167 & 98 & 148 & 104 \\
\hline Output & $K_{17}$ & 127 & 111 & 186 & 111 & 62 & 132 & 35 & 230 & 184 & 23 & 199 & 252 & 186 & 75 & 227 & 149 \\
\hline Output & ciphertext & 224 & 31 & 182 & 10 & 12 & 255 & 84 & 70 & 127 & 13 & 89 & 249 & 9 & 57 & 165 & 220 \\
\hline
\end{tabular}


INTERNATIONAL JOURNAL Of ENGINEERING TECHNOLOGIES-IJET

Şahin, et al, Vol.6, No.2, 2020

\subsection{Digital Image Encryption/ Decryption}

The performance of software implementation of SAFER+ is also tested with image encryption/ decryption using the same procedure as in the data block previously explained. A two dimentional (gray level) image is encrypted using SAFER+ as shown in the Fig. 8, where the encryption takes a time of $2.573912 \mathrm{sec}$, and the decryption time is $2.591723 \mathrm{sec}$. The decryption attempt of the image with wrong key is depicted in the same figure. where the result is an absolute noise. The use of correct symmetrical key regenerates the original image by a successful decryption process. The image histogram distributions before and after encryption are presented in the Fig. 9. A new procedure is developed that encrypts/ decrypts each pixel providing an additional security measure; in that if the algorithm senses three consecutive attack attempts by fake keys, the image is immediately destroyed with irreversible procedure as illustrated in the Fig. 10.

\section{Conclusion}

SAFER+ algorithm is a fast cipher system that draws attention with its capability and performance in Bluetooth communication. The implementation of the algorithm with the option of 128 bits key length is performed using MATLAB. The results show a good performance in terms of encryption/ decryption speed compared to the standard algorithm AES. The software implementation of the nonlinears functions which has been problematic, is resolved by the ELF procedure. The simulation of encrypted image offered a high speed, inexpensive tool that can be used by small companies or for personal use. The implementation of this algorithm in hardware such as in FPGA can offer a further improvement in terms of speed which will enable this cipher standard to take place among the fastest and most secure existing standard.

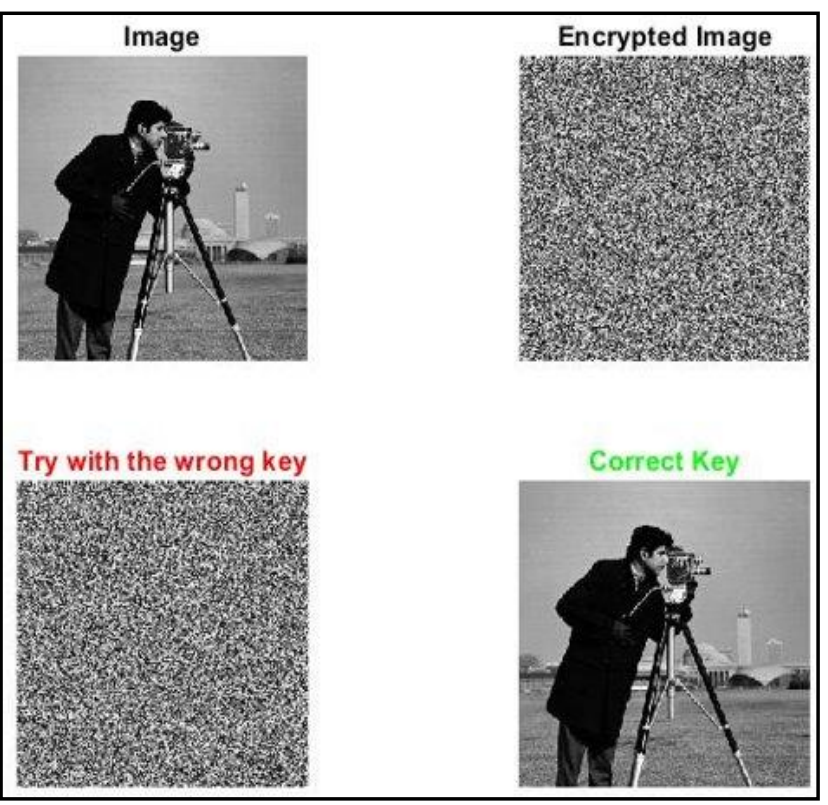

Figure 8. Image Encryption and Decryption Results

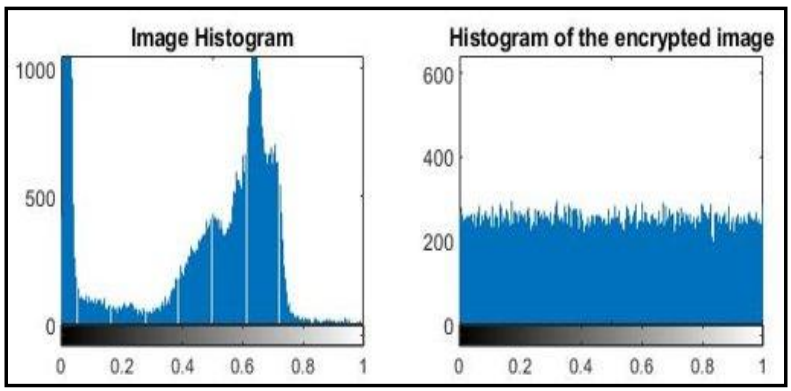

Figure 9. Image Histograms

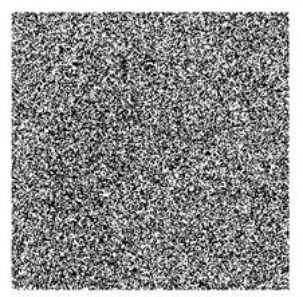

Figure 10. Result of additional security measures

\section{References}

[1] N. Alhalafi, and P. Veeraraghavan, "Privacy and Security Challenges and Solutions in IOT: A review", Int. Conf. on 
INTERNATIONAL JOURNAL of ENGINEERING TECHNOLOGIES-IJET

Şahin, et al, Vol.6, No.2, 2020

Smart Power \& Internet Energy Systems, Melbourne, Australia, pp 1-5, 25 April 2019.

[2] V. Shree, Z. Antony, and N. Jayapandia," Enhanced Data Security Architecture in Enterprise Network", Springer Int. Conf. on Computer Networks, Big data and IoT, India, pp. 857-864, Dec. 2019.

[3] M. B. Yassein, et al., “Comprehensive Study of Symmetric Key and Asymmetric Key Encryption Algorithm", Int. Conf. on Engineering and Technology, Antalya, Turkey, 2017.

[4] W.N.A. Ruzai, M.R.K. Ariffin, M.A. Asbullah, Z. Mahad, and A. Nawaw, "On the Improvement Attack Upon Some Variants of RSA Cryptosystem via the Continued Fractions Method”, IEEE Access, Vol. 8, pp.1-10, April, 2020.

[5] A. Alabaichi, F. Ahmed, and R. Mahmod, "Security Analysis of Blowfish algorithm”, IEEE Second Int. Conf. on Informatics \& Applications (ICIA), Poland, pp. 12-18, 23 Sept. 2013.

[6] S. Mukherjee, D. Ganguly, and S. Naskar, "A New Generation Cryptographic Technique", International Journal of Computer Theory and Engineering, Vol. 1, No. 3, pp. 284287, August 2009.

[7] G. Mustafa, et al., "A Review of Data Security and Cryptographic Techniques in IoT based devices", The $2^{\text {nd }}$ Int.

Conf. on Future Networks \& Distributed Systems, Amman, Jordan, June 26-27, 2018.

[8] D. L. Kumar, A. R. Reddy, and S. A. K. Jilani, "Implementation of 128-bit AES algorithm in MATLAB",

International Journal of Engineering Trends and Technology, Vol. 33 No. 3, pp. 126-129, March 2016.

[9] S. Chandra, et al., "A comparative survey of symmetric and asymmetric key cryptography", IEEE Int. Conf. on Electronics, Communication and Computational Engineering, India, pp. 83-93, 2014.

[10] J. Messy, H. Kh and K. Kuregian, Nomination of SAFER+ as a candidate algorithm for the AES, 1998. http://csrc.nist.gov/archive/aes/round1.

[11] B. J. Babu, D. K. M. Tech, and R. V. V. Krishna, "Design of SAFER+ Encryption Algorithm for Bluetooth Transmission", International Journal of Innovative Technology and Research, Vol. No.3, Issue 1, pp. 1864 1867, Dec. 2015.

[12] A. Schubert, and W. Anheier, "Efficient VLSİ implementation of modern symmetric block ciphers", $6^{\text {th }}$ IEEE Int. Conf. on Electronics, Circuits and Systems, Vol. 2, Cyprus, pp. 757-760, Sept. 1999,

[13] M. K. Mahmood, F. M. Al-Naima, "Developing a MultiLayer Strategy for Securing Control Systems of Oil Refineries", Wireless Sensor Network, Vol. 2, pp. 520-527, July 2010.

[14] M.K. Mahmood, L. S. Abdulla, A. H. Mohsin, and H. A. Abdulla, “ MATLAB Implementation of 128-key length SAFER+ Cipher System", Int. Journal of Engineering Research and Application, Vol. 7, İssue 2, pp. 49-55, Feb. 2017. 